\title{
Antibiotic Production by Dermatophyte Fungi
}

\author{
By NAGWAN YOUSSEF, C. H. E. WYBORN AND G. HOLT \\ Life Sciences, Polytechnic of Central London, \\ 115 New Cavendish Street, London W1M 8JS
}

W. C. NOBLE AND YVONNE M. CLAYTON

Institute of Dermatology, St John's Hospital for Diseases of the Skin, London E9 6BX

(Received 16 September 1977)

\begin{abstract}
Thirty-two clinical isolates of anthropophilic dermatophytes were examined for their capacity to produce antibiotics in liquid culture and on human stratum corneum in vitro. Antibiotics were detected and classified using agar diffusion bioassays and chromatographic analysis. Twenty-four isolates produced antibiotic substances in liquid culture filtrates; some strains produced more than one antibiotic. Only four isolates produced detectable levels of antibiotics when grown on stratum corneum unless an artificial sweat mixture was used as a nutrient supplement, when the number rose to 11 . Representatives of all species studied produced benzyl penicillin-like substances. Some Trichophyton isolates also produced streptomycin-like antibiotics, a characteristic previously unrecorded for eukaryotic organisms. Other antibiotics, which apart from azalomycin $\mathrm{F}$ could not be properly classified, were produced by Epidermophyton floccosum. Antibiotic production occurred over the normal skin temperature range but sometimes the type of antibiotic produced and the frequency of detection appeared to be influenced by the incubation temperature.
\end{abstract}

\section{INTRODUCTION}

Although the production of antibiotics by dermatophyte fungi has long been known, there have been few systematic studies of either production or the ecological effects of production. The earliest reports of the production of antibacterial substances by dermatophytes appear to be those of Nakamura (1932) and Honda (1936). Peck \& Hewitt (1945) believed the antibacterial product to resemble penicillin and Uri, Valu \& Békési (1963) later demonstrated the production of benzyl penicillin by Trichophyton mentagrophytes. In a study of Epidermophyton floccosum, Nishio (1958) detected antibiotics in culture filtrates using column chromatography: penicillin-like and actinomycin-like antibiotics were indicated. Cole (1966) investigated the formation of 6-aminopenicillanic acid and penicillin by various fungi, and concluded that both substances were produced by E. floccosum. Geophilic dermatophytes were shown to produce antibiotics by Gip \& Palsson $(1970 a, b)$, who found that Microsporum cookei inhibited Staphylococcus aureus strains both resistant and sensitive to benzyl penicillin. Wallerstrom $(1967,1968,1969)$ concentrated on E. floccosum and related the 'epidermophyton factor' (EPF) to steroid antibiotics like fucidin. He also demonstrated that some E. floccosum strains produce penicillin.

If antibiotics are produced in vivo two effects might be noticed. One is the sensitization of the infected person towards the antibiotic produced. There is some epidemiological evidence that this occurs (Bolgar et al., 1960; Schuppli, 1962; Blum \& De Weck, 1966). The other effect might be a shift in the local bacterial flora towards antibiotic resistance. Wallerstrom (1968) was able to demonstrate that lesions infected with E. floccosum had a microflora 
more resistant to EPF and penicillins than did the control samples (from persons with clinical lesions, but from whom no fungus was grown). In a study of experimental dermatophyte infection in human volunteers, Bibel \& Le Brun (1975) found that the proportion of penicillin-resistant cocci at the site of infection increased during the course - of infection, but decreased rapidly after the fungi became absent from the lesion.

In a preliminary study, Noble \& Clayton (unpublished observations) investigated an unselected series of 98 patients with groin lesions resembling tinea cruris. Dermatophyte fungi were cultured as part of the routine clinical investigation; samples were obtained by scraping affected stratum corneum and were cultured on Sabouraud's agar. The bacterial flora was sampled using a cotton swab moistened in broth, and held in Stuart's Transport Medium until inoculated on to blood agar. Bacterial isolates were characterized as Gram-negative rods, S. aureus, other Micrococcaceae or diphtheroids. All Gram-positive species were tested for resistance to penicillin, tetracycline, neomycin, erythromycin, gentamicin and clindamycin using Oxoid Multodiscs. Gram-negative bacilli were tested for resistance to ampicillin, sulphonamide, sulphomyxin sodium, colistin sulphomethate sodium, gentamicin, nitrofurantoin and kanamycin. Clear-cut results were not obtained; $57 \%$ of the 42 patients from whom fungus was cultured carried penicillin-resistant cocci compared with only $38 \%$ of the 56 patients from whom no fungus was cultured. However, this may not be surprising since natural variations among wild-type isolates of fungi producing penicillin have been reported for the Penicillium notatum group (Raper, Alexander \& Coghill, 1944) and Aspergillus nidulans (Holt \& Macdonald, 1968). The probability was that not all of the dermatophytes investigated in the initial study were antibiotic producers. To test this hypothesis it was decided to investigate a number of clinical isolates for their ability to produce antibiotics in vitro, both in a synthetic fermentation medium and on strips of human stratum corneum.

\section{METHODS}

Dermatophyte cultures. Thirty-two anthropophilic isolates cultured from tinea lesions at St John's Hospital for Diseases of the Skin were studied. Eight isolates were characterized as Trichophyton mentagrophytes, 11 as Trichophyton rubrum and 13 as Epidermophyton floccosum. Isolates were allocated consecutive domestic code numbers. All cultures were maintained on Sabouraud agar (Oxoid) slopes in $30 \mathrm{ml}$ Universal bottles.

Bacteria used in bioassays. These were: Bacillus subtilis (NCTC 8236); Escherichia coli (NCIB 10224), resistant to streptomycin; Escherichia coli ( $\mathrm{NClB} 86$ ), sensitive to streptomycin; Staphylococcus aureus, resistant to penicillin (a penicillinase producer which was resistant to $10 \mathrm{u}$. benzyl penicillin when tested by the disc method); and Staphylococcus aureus, sensitive to penicillin. The two $S$. aureus strains were of the same origin, the sensitive variant being derived from the resistant strain by loss of the penicillinase plasmid (Noble, 1977).

Antibiotic production medium. As penicillin was of particular interest in the study, a fermentation unit medium (FUM) specifically designed for benzyl penicillin production was used (Holt \& Macdcnald, 1968). A mycelial inoculum of the test fungus was added to $25 \mathrm{ml}$ FUM in a $100 \mathrm{ml}$ polypropylene conical flask. Three flasks were inoculated for each strain. The inoculum consisted of mycelium grown for $6 \mathrm{~d}$ at $30{ }^{\circ} \mathrm{C}$ in a $30 \mathrm{ml}$ Universal bottle containing $3 \mathrm{ml}$ Sabouraud broth (Oxoid). Flasks were incubated for $7 \mathrm{~d}$ on a rotary shaker ( $200 \mathrm{rev} . \mathrm{min}^{-1}$ and $5 \mathrm{~cm}$ eccentric throw) at 26,30 and $33^{\circ} \mathrm{C}$. Broth from the shake flasks was filtered through Whatman no. 1 filter paper, and the filtrates were used in bioassays and chromatography.

Antibiotic bioassay. Antibiotics were detected in culture filtrates using a standard agar-plate diffusion technique with or without penicillinase $(\mathrm{BDH})$ incorporated at a concentration of $80 \mathrm{u.} \mathrm{ml} \mathrm{m}^{-1}$ (Cole, Holt \& Macdonald, 1976). Diagnostic Sensitivity Test Agar (DSTA, Oxoid) was seeded with B. subtilis spores. Assay plates were incubated at $37^{\circ} \mathrm{C}$ for $16 \mathrm{~h}$. Culture filtrates which produced inhibition zones were further analysed by paper chromatography.

Chromatography and bioautography. Crude concentrates were prepared by acetone extraction of filtrates and divided into two parts, one part being treated with penicillinase $\left(80 \mathrm{u} . \mathrm{ml}^{-1}\right)$ to inactivate benzyl penicillin-like antibiotics. Antibiotics were characterized using the system devised by Betina (1964) in which the patterns of $R_{F}$ values, obtained when different solvents are used in an ascending paper chromatographic system, are characteristic of particular antibiotics. A bioautographic procedure was used to visualize the distance run by the antibiotic. Dried chromatograms were applied to the surface of assay plates of DSTA seeded with $B$. subtilis spores. Plates were held at $4{ }^{\circ} \mathrm{C}$ for $3 \mathrm{~h}$, followed by $16 \mathrm{~h}$ incubation at $37^{\circ} \mathrm{C}$. 
Standard antibiotic preparations. Benzyl penicillin and streptomycin sulphate (Glaxo) were used for comparison in bioassays and chromatography.

In vitro growth of dermatophytes on human stratum corneum. The technique devised by Knight (1972) to study dermatophyte growth on stratum corneum was modified to permit detection of antibiotics excreted by the fungi. Layers of stratum corneum were removed from healthy skin on human forearms by applying a $4 \times 2 \mathrm{~cm}$ strip of translucent adhesive tape ('Steridrape', Minnesota Mining and Manufacturing Co., St Paul, Minnesota, U.S.A.). Each strip was applied several times to the skin until it no longer adhered. Strips were fixed, stratum corneum uppermost, on to microscope slides resting on glass rods in Petri dishes and sterilized by ethylene oxide at least $3 \mathrm{~d}$ before inoculation.

For each isolate, the influence on antibiotic production of synthetic sweat mixture, a synthetic sebum preparation (Murphy, 1975) and a combination of both in equal proportions was investigated using the Steridrape technique. In each case, $5 \mu 1$ of the appropriate sterile mixture or sterile distilled water were spread across the skin strips and allowed to dry. Sterile distilled water $(8 \mathrm{ml})$ was placed in the bottom of each dish. Each inoculum consisted of $5 \mu \mathrm{l}$ of spore suspension from a fungal isolate spread across the strip. Duplicate inoculated and control strips were incubated for $7 \mathrm{~d}$ at $30^{\circ} \mathrm{C}$. In addition, inoculated and control strips with sweat supplement or distilled water were incubated at 26 and $33{ }^{\circ} \mathrm{C}$. Antibiotic activity was detected by the same procedure described for bioautography, the skin strip substituting for the chromatogram.

Where antibiotic production was positively demonstrated following fungal growth on stratum corneum, bioassays were performed to characterize the nature of the antibiotics more precisely. These bioassays involved the use of : (i) B. subtilis with and without penicillinase in the medium; (ii) penicillin-resistant and -sensitive isolates of $S$. aureus; (iii) streptomycin-resistant and -sensitive strains of $E$. coli.

\section{RESULTS AND DISCUSSION}

Using the classification of Betina (1964), at least three different antibiotics were recognized in culture filtrates of many of the 32 dermatophyte fungi examined. Figure 1 shows the summarized chromatograms for antibiotics produced by three representative isolates. For strains 3 and 9, the patterns of $R_{F}$ values corresponded to those observed with known standards of benzyl penicillin and streptomycin sulphate respectively. The antibiotic activity of strain 3 was not detected after penicillinase treatment of the culture filtrates. Additionally, a penicillin-resistant strain of $S$. aureus was not inhibited by the antibiotic whereas a sensitive isolate was. After penicillinase treatment of the culture filtrates of strain 9 , the antibiotic produced inhibited a strain of Esch. coli sensitive to streptomycin but not a resistant one. The $R_{F}$ values of the antibiotic produced by strain 1 were similar to the published data for azalomycin $F$, but no authentic sample was available for comparison.

The antibiotics produced by the dermatophytes grown in liquid culture and on stratum corneum in vitro at different incubation temperatures are shown in Table 1 . The capacity of anthropophilic dermatophytes to produce a range of antibiotics, some therapeutic, is obviously a common but probably not a universal characteristic. Some $75 \%$ of the isolates investigated produced antibiotics when grown in liquid culture, including all $8 \mathrm{~T}$. mentagrophytes isolates, 7 of the $11 \mathrm{~T}$. rubrum isolates and 9 of the $13 \mathrm{E}$. floccosum isolates. For Trichophyton isolates, the characterized antibiotics were benzyl penicillin-like or streptomycin-like. For E. floccosum, apart from benzyl penicillin-like and azalomycin F-like antibiotics, other antibacterial substances were produced by several isolates. Most of these fell within group 4 of Betina's classification, which includes azalomycin $\mathrm{F}$ and amphotericin B amongst others, but could not be characterized further by the patterns of $R_{F}$ values. Although many isolates were able to produce more than one antibiotic, it is possible that the acetone extraction procedure chosen to concentrate the antibiotics from culture filtrates selected certain antibiotics. Others may have been present, but not concentrated. This is supported by the bioassay results for $T$. mentagrophytes isolates grown on stratum corneum where antibacterial substances other than those resembling benzyl penicillin and streptomycin were apparent. The greater frequency of detection of benzyl penicillin-like substances in liquid culture reflects the choice of the medium which is specially designed for benzyl penicillin synthesis. It may not be significant that detection of other antibiotics in culture filtrates was only demonstrated for penicillin-producing isolates. It could well be that a 


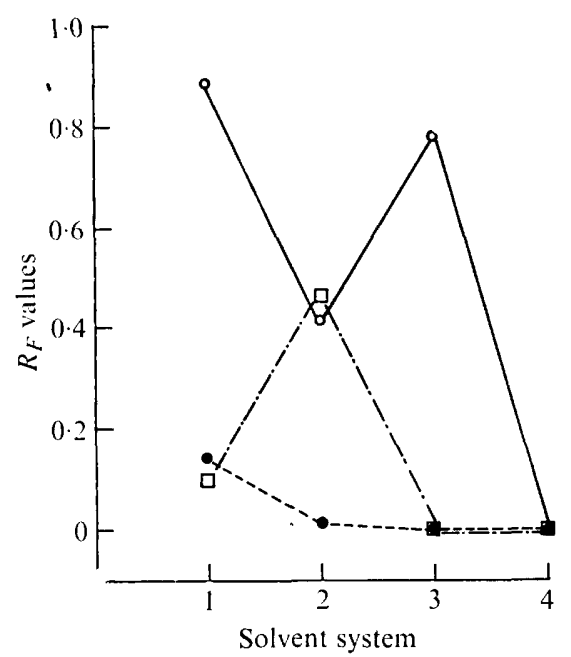

Fig. 1. Summarized chromatograms of antibiotics extracted with acetone from culture filtrates of three representative dermatophyte isolates:,$T$. mentagrophytes strain 9 (streptomycin-like); $\bigcirc, T$. rubrum strain 3 (benzyl penicillin-like); $\square$, E. floccosum strain 1 (azalomycin F-like). Solvents: 1 , distilled water; $2, n$-butanol saturated with water; 3 , ethyl acetate saturated with water; 4 , benzene saturated with water.

medium designed, for example, for streptomycin production might result in enhanced streptomycin detection. However it is noteworthy that FUM was able to support the production of a variety of antibiotics.

The detection of streptomycin-like substances in culture filtrates of over half of the Trichophyton isolates investigated, as well as on stratum corneum during growth of two $T$. mentagrophytes strains, was totally unexpected. If substantiated, this would appear to be the first record of streptomycin production, or similar aminoglycosides, by eukaryotic organisms.

The reduced frequency of antibiotic detection when dermatophytes were grown on stratum corneum in vitro was apparently not due to reduced diffusion rates. Standard preparations of benzyl penicillin and streptomycin sulphate diffused easily across both the stratum corneum and Steridrape, with and without synthetic sweat and sebum preparations, although the diffusion rate was greater for penicillin than streptomycin. The reduced frequency of detection may reflect either the poorer nutrient status of the stratum corneum compared to FUM, or complexing of antibiotics with non-diffusible substances in the stratum corneum.

The synthetic sebum and sweat preparations used as supplements represent fat-soluble and water-soluble components of skin secretions, respectively (Murphy, 1975). The sebum preparation appeared to be inhibitory to both antibiotic production and mycelial growth, the latter being very sparse. In contrast, the sweat supplement enhanced mycelial growth and antibiotic production on the stratum corneum and this probably reflects the increased nutrient status of the system. Such effects could be of ecological significance as areas of skin with high humidity and excessive sweating are often the foci of dermatophyte infections in adults. According to Noble \& Somerville (1974) the skin temperature is maintained between 30 and $33{ }^{\circ} \mathrm{C}$ in a comfortable environment, though crural lesions must more closely approach $37^{\circ} \mathrm{C}$. The temperature range appears adequate for dermatophytes to produce antibiotics when growing on skin (cf. Table 1).

There is little evidence that dermatophytes make the skin totally unsuitable for multiplication of indigenous bacteria. On the contrary, dermatophytes may facilitate colonization by $S$. aureus which subsequently contributes to the appearance of symptoms (Marples \& 
Table 1. Antibiotics detected when dermatophytes were grown in vitro either in fermentation unit medium (FUM) or on stratum corneum (SC) with and without synthetic supplements

\begin{tabular}{|c|c|c|c|c|c|c|c|c|}
\hline \multirow{2}{*}{$\begin{array}{l}\text { Domestic } \\
\text { code number }\end{array}$} & \multicolumn{3}{|c|}{ FUM at: } & \multicolumn{3}{|c|}{ SC+ synthetic sweat at: } & \multirow{2}{*}{$\begin{array}{c}\text { SC alone } \\
30^{\circ} \mathrm{C}\end{array}$} & \multirow{2}{*}{$\begin{array}{r}\mathrm{SC}+\mathrm{Sw} \\
\text { sebun } \\
\text { mixtur } \\
\text { at } 30^{\circ}\end{array}$} \\
\hline & $26^{\circ} \mathrm{C}$ & $30^{\circ} \mathrm{C}$ & $33^{\circ} \mathrm{C}$ & $26^{\circ} \mathrm{C}$ & $30^{\circ} \mathrm{C}$ & $33^{\circ} \mathrm{C}$ & & \\
\hline \multicolumn{9}{|c|}{ T. rubrum } \\
\hline 2 & $\mathbf{P}$ & PS & PS & - & - & - & - & - \\
\hline 3 & $\mathbf{P}$ & $P$ & $\mathbf{P}$ & - & $\mathbf{P}$ & $\mathbf{P}$ & $\mathbf{P}$ & $\mathbf{P}$ \\
\hline 4 & $\mathrm{P}$ & PŚ & PS & - & $\mathbf{P}$ & $P$ & - & - \\
\hline 5 & $\mathbf{p}$ & $\mathbf{P}$ & $\mathbf{P}$ & - & $\mathbf{P}$ & - & - & - \\
\hline 18 & - & PS & s & - & - & - & - & . \\
\hline 19 & $\mathbf{P}$ & - & - & - & - & - & - & - \\
\hline 24 & $\mathbf{P}$ & PS & $\mathbf{s}$ & - & - & - & - & - \\
\hline \multicolumn{9}{|c|}{ T. mentagrophytes } \\
\hline 7 & $\mathbf{P}$ & $\mathbf{P}$ & $\mathbf{P}$ & - & $\mathbf{P}$ & $\mathbf{P}$ & $\mathbf{P}$ & $\mathbf{P}$ \\
\hline 8 & $\mathbf{P}$ & $\mathbf{P}$ & $\mathbf{P}$ & - & - & - & - & - \\
\hline 9 & PS & PS & s & - & $\mathbf{s}$ & s & - & - \\
\hline 10 & PS & PS & $\mathbf{s}$ & - & $\mathrm{U}$ & $\mathbf{U}$ & - & - \\
\hline 25 & PS & PS & s & - & - & - & - & - \\
\hline 26 & PS & PS & s & - & - & - & - & - \\
\hline 27 & $\mathbf{P}$ & PS & PS & - & $\mathbf{U}$ & $U$ & - & - \\
\hline 38 & $P$ & PS & PS & $\mathbf{S}$ & s & $\mathbf{s}$ & $\mathbf{s}$ & S \\
\hline \multicolumn{9}{|c|}{ E. floccosum } \\
\hline 1 & $\mathbf{P A}$ & $\mathbf{P A}$ & $\mathbf{P A}$ & - & - & - & - & _ \\
\hline 14 & PA & PA & $\mathbf{P A}$ & - & - & - & - & - \\
\hline 16 & PU & PU & $\mathrm{U}$ & - & - & - & - & 7 \\
\hline 17 & PU & PU & PU & - & - & - & - & - \\
\hline 32 & PU & PU & $\mathbf{U}$ & - & $\mathrm{U}$ & $\mathbf{U}$ & $u$ & U \\
\hline 34 & PU & PU & PU & - & $\mathrm{U}$ & - & - & - \\
\hline 35 & PU & PU & PU & - & $\mathbf{U}$ & $\mathrm{U}$ & - & - \\
\hline 36 & PU & PU & PU & - & - & - & - & . \\
\hline 37 & PU & PU & PU & - & - & - & - & - \\
\hline
\end{tabular}

P, Benzyl penicillin-like; acetone extracts of culture filtrates with $R_{F}$ values characteristic of benzyl penicillin; antibiotic activity destroyed by penillinase treatment; active against strains of $S$. aureus sensitive to benzyl penicillin, but not against resistant strains.

s, Streptomycin-like; acetone extracts of culture filtrates with $R_{F}$ values characteristic of streptomycin; active against Esch. coli ( $\mathrm{NCIB} 86$ ) sensitive to streptomycin, but not against Esch. coli (NCIB 10224) resistant to streptomycin.

A, Azalomycin F-like; acetone extracts of culture filtrates with $R_{F}$ values resembling published data for azalomycin F; active against bacteria used in bioassays except Esch. coli strains.

$\mathrm{U}$, Uncharacterized; acetone extracts of culture filtrates with $R_{F}$ values not resembling published data for antibiotics; antibiotic activity not destroyed by penicillinase treatment; active against all bacteria used in bioassays.

-, No antibiotics detected in amounts inhibitory to B. subtilis (NCTC 8236).

Bailey, 1957). Allen \& Taplin (1974) suggested that the high rate of penicillin resistance amongst staphylococci in a human population in Panama, in the apparent absence of therapeutic penicillin, may be due to naturally occurring penicillin in the environment. Dermatophyte infections could provide an appropriate natural source. There are indications that cocci associated with tinea lesions do have a higher prevalence of antibiotic resistance (Wallerstrom, 1968; Bibel \& Le Brun, 1975). Although it is not possible to extrapolate the results from experiments in vitro to conditions in vivo, this study suggests that genetic variability in the capacity of dermatophytes to produce antibiotics, together with fluctuations in temperature and skin secretions at the site of infection, might result in considerable variation in the antibiotic resistance of bacteria associated with tinea lesions. This would explain the poor correlation between the antibiotic resistance of bacteria and the fungal 
flora of tinea cruris lesions noted by Noble \& Clayton (unpublished observations, see Introduction).

It is necessary to assess the antibiotic production of the fungus while growing in skin in relation to the antibiotic resistance of the bacterial flora. Only Wallerstrom (1968) has attempted to do this. It would be interesting to know the antibiotic-producing potential of the strain used by Bibel \& Le Brun (1975) in their experiments. Investigation of the antibiotic production by zoophilic dermatophytes could also prove rewarding, since Smith \& Marples (1964) found that hedgehogs infected by dermatophytes carried a high proportion of penicillin-resistant $S$. aureus strains in their microflora.

Published studies on skin as a microbial habitat, and interactions between the constituents of the microflora in vivo, are still few. Selwyn (1975) has shown how staphylococci may produce antibiotics active against other cocci, and Noble (1977) has demonstrated that genetic variants may appear on skin in response to some unknown ecological pressure. Characterization of the different antibiotics synthesized by dermatophytes, conditions under which they may be produced in the living host, and possible effects on the resident microflora are now under investigation. It can be expected that such studies on the skin flora of man and other mammals will yield valuable results for microbial ecology in general.

We wish to thank the Government of the Arab Republic of Egypt for providing a mission study award to one of us (N.Y.). Some of the work reported here forms part of a submission to the C.N.A.A. for the degree of Doctor of Philosophy. We are grateful to the National Heart Hospital for ethylene oxide sterilization facilities.

\section{REFERENCES}

Allen, A. M. \& Taplin, D. (1974), Skin infections in eastern Panama. Survey of two representative communities. American Journal of Tropical Medicine and Hygiene 23, 950-956.

Betina, V. (1964). A systematic analysis of antibiotics using paper chromatography. Journal of Chromatography 15, 379-392.

Bibel, D. J. \& LE BRUN, J. R. (1975). Effect of experimental dermatophyte infection on cutaneous flora. Journal of Investigative Dermatology 64, 119-123.

Blum, G. \& De Weck, A. L. (1966). Über den Zusammenhang von Dermatomykosen mit der Penicillinüberempfindlichkeit. Dermatologica 133, 461-472.

Bolgar, E., Fehér, E., Török, H. \& Rajka, E. (1960). Über Penicillinallergie. Hautarzt 11, 254 258.

Cole, D. S., Holt, G. \& Macdonald, K. D. (1976). Relationship of the genetic determination of impaired penicillin production in naturally occurring strains to that in induced mutants of Aspergillus nidulans. Journal of General Microbiology 96, 423-426.

Cole, M. (1966). Formation of 6-aminopenicillanic acid, penicillins and penicillin acylase by various fungi. Applied Microbiology 14, 88-104.

Gip, L. \& Palsson, G. (1970a). Production of antibiotics by geophilic dermatophytes. I. Screening test of antibacterial activity. Mykosen 13, 397400 .

Gip, L. \& Palsson, G. (1970 b). Production of antibiotics by geophilic dermatophytes. II. Antibiotic spectrum of crude filtrates. Mykosen 13, 521523.

Holt, G. \& Macdonald, K. D. (1968). Penicillin production and its mode of inheritance in Aspergillus nidulans. Antonie van Leeuwenhoek 34, 409-416.

Honda, S. (1936). Über das antagonistische Phaenomen zwischen verschiedenen pathogenen Schimmelpilzen und Bakterien. Japanese Journal of Dermatology 39, 97-98.

KNIGHT, A. G. (1972). Culture of dermatophytes upon stratum corneum. Journal of Investigative Dermatology 59, 427-431.

Marples, M. J. \& BaIley, M. J. (1957). A search for the presence of pathogenic bacteria and fungi in the interdigital spaces of the foot. British Journal of Dermatology 69, 379-388.

MurPhy, C. T. (1975). Nutrient materials and the growth of bacteria on human skin. Transactions. St John's Hospital Dermatological Society 61, 51-57.

NAKamura, T. (1932). Über die antagonistische Wirkung des Trichophytonpilzes gegen Heubazillus. Japanese Journal of Dermatology 32, 126.

NiSHIO, K. (1958). Epidermophyton inguinale in der Antibioticum-Forschung. Japanese Journal of Dermatology 20, 221-239.

Noble, W. C. (1977). Variation in the prevalence of antibiotic resistance of Staphylococcus aureus from human skin and nares. Journal of General Microbiology 98, 125-132.

Noble, W. C. \& Somerville, D. A. (1974). Microbiology of Human Skin. London: W. B. Saunders. 
Peck, S. M. \& HewitT, W. L. (1945). The production of an antibiotic substance similar to penicillin by pathogenic fungi. Public Health Reports 60, 148-193.

Raper, K. B., Alexander, D. F. \& Coghill, R. D. (1944). Penicillin. II. Natural variation and penicillin production in Penicillium notatum and allied species. Journal of Bacteriology 48, 639659.

Schuppli, R. (1962). Über den Zusammenhang zwischen Penicillinallergie und Pilzinfektionen. Deutsche medizinische Wochenschrift 87, 333-335.

SELWYN, S. (1975). Natural antibiosis among skin bacteria as a primary defence against infection. British Journal of Dermatology 93, 487-493.

Smith, J. M. B. \& Marples, M. J. (1964). A natural reservoir of penicillin-resistant strains of Staphylococcus aureus. Nature, London 201, 844.
URI, J., VAlu, G. \& BÉkÉsi, I. (1963). Production of 6-a.p.a. by dermatophytes. Nature, London 200 , 896-897.

Wallerstrom, A. (1967). Production of antibiotics by Epidermophyton floccosum. 1. The antibiotic spectrum of crude filtrates. Acta pathologica et microbiologica scandinavica 71, 287-295.

WAllerstrom, A. (1968). Production of antibiotics by Epidermophyton floccosum. 2. Microflora in Epidermophyton-infected skin and its resistance to antibiotics produced by the fungus. Acta pathologica et microbiologica scandinavica 74, 531542.

Wallerstrom, A. (1969). Production of antibiotics by Epidermophyton floccosum. 3. Course of fermentation and some properties of the epidermophyton factor (E.P.F.). Acta pathologica et microbiologica scandinavica 75, 435-446. 